\title{
Gastrointestinal Bleeding and Endoscopic Outcomes in Patients with SARS-CoV-2
}

\author{
Faruq Pradhan ${ }^{1,2}$ and Yasmin Alishahi ${ }^{2}$ \\ ${ }^{1}$ Department of Gastroenterology, Banner University Medical Center Phoenix, Phoenix, AZ, ${ }^{2}$ Department of Gastroenterology, Phoenix \\ VA Health Care System, Phoenix, AZ, USA
}

Over the past year, the novel coronavirus has been a topic of significant research. Multiple gastroenterological symptoms have been associated with this infection, in addition to the well-established pulmonary presentations. Gastrointestinal bleeding can be a complication of infection by severe acute respiratory syndrome coronavirus-2, which can be exacerbated by the anticoagulants used to treat its thrombotic sequelae. We describe the clinical cases of four patients infected with the novel coronavirus, with significant upper gastrointestinal bleeding requiring endoscopic visualization, along with their clinical outcomes. Clin Endosc 2021;54:428-431

Key Words: Coronavirus; Endoscopy; Hemorrhage

\section{INTRODUCTION}

The global pandemic due to severe acute respiratory syndrome coronavirus-2 (SARS-CoV-2) has led to numerous deaths worldwide. The virus, referred to as coronavirus disease (COVID-19), has multisystem involvement, including gastrointestinal (GI) manifestations such as diarrhea, nausea, and vomiting. GI bleeding has also been noted in infected patients, and several recent studies have reported favorable clinical outcomes in the conservative management of patients with COVID-19. ${ }^{1,2}$ We present four recent cases (over a 4-week period) of patients with COVID-19, who required urgent endoscopy owing to clinically significant upper GI bleeding, and describe the endoscopic interventions provided and their clinical outcomes.

Received: September 7, 2020 Revised: October 7, 2020

Accepted: October 7, 2020

Correspondence: Faruq Pradhan

Department of Gastroenterology, Banner University Medical Center Phoenix, 1111 E. McDowell Road, Phoenix, AZ 85006, USA

Tel: +1-602-839-2000, Fax: +1-602-521-5334, E-mail: faruq.pradhan@bannerhealth.com

ORCID: https://orcid.org/0000-0002-5249-188X

(c) This is an Open Access article distributed under the terms of the Creative Commons Attribution Non-Commercial License (http://creativecommons.org/ licenses/by-nc/3.0) which permits unrestricted non-commercial use, distribution, and reproduction in any medium, provided the original work is properly cited.

\section{CASE REPORTS}

\section{Case 1}

A 69-year-old Caucasian man with a medical history of hypertension, depression, obesity, and obstructive sleep apnea, presented to the hospital with complaints of fatigue, shortness of breath, cough, and diarrhea for 1 week. He was found to have COVID-19 at presentation, confirmed using reverse transcription polymerase chain reaction (RT-PCR). At the time of presentation, the patient was in acute hypoxic respiratory failure requiring intubation. He was started on dexamethasone and convalescent plasma as therapy for COVID-19, along with ceftriaxone and azithromycin for community-acquired pneumonia. On hospital day 3 , the patient had a new onset of bloody output from his orogastric tube, along with melena and an increase in vasopressor requirements. There was no known history of liver disease or the use of non-steroidal anti-inflammatory drugs (NSAIDs) or antiplatelet agents. Upper endoscopy on hospital day 4 identified a large, 15-20 $\mathrm{cm}$, organized clot in the stomach that was not amenable to removal, despite vigorous irrigation and suctioning (Fig. 1A). Non-bleeding, superficial erosions were seen along the greater curvature, possibly due to orogastric tube trauma. There was no evidence of active bleeding. Although the patient had no further episodes of bleeding after conservative management, he ultimately died on hospital day 12 owing to complications 

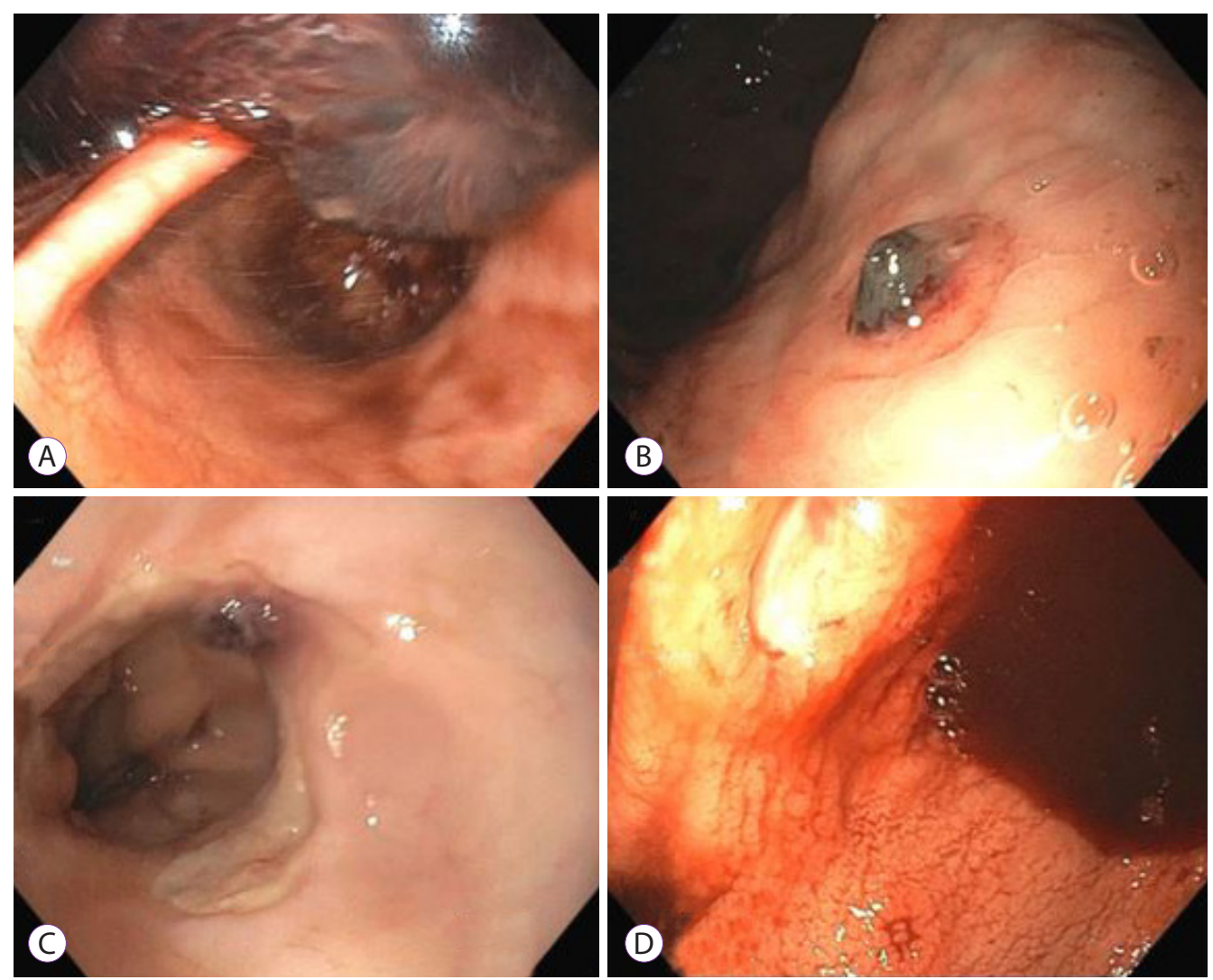

Fig. 1. Summary of endoscopic findings: (A) retroflexed view on upper endoscopy (case 1) with a large clot obstructing view, (B) the greater curvature of the stomach with a visible vessel (case 2), (C) a visible vessel with ulceration in the distal esophagus (case 3), and (D) gastric antrum visualized on upper endoscopy (case 4) with one (of many) ulcers.

of hypoxic respiratory failure and cardiogenic shock.

\section{Case 2}

A 68-year-old African-American man with a medical history of decompensated cirrhosis due to hepatitis $\mathrm{C}$ (complicated by prior variceal bleed, ascites, and hepatic encephalopathy), end-stage renal disease (on hemodialysis), atrial fibrillation (on aspirin), and prior deep vein thrombosis (completely treated with a course of rivaroxaban), presented to the hospital with a 1 -week history of progressive confusion. He was found to have COVID-19 on presentation by RT-PCR testing. He was initially admitted to the intensive care unit (ICU) for urgent dialysis to correct hyperkalemia but was transferred to the medical floor the following day. He was started on dexamethasone for COVID-19 when he was admitted, which was terminated when he was transferred out of the ICU. On hospital day 5, the patient had an episode of hematemesis in the night and passed melenic stools the next morning. He had an episode of syncope while he was on hemodialysis, with hypotension and worsening confusion. He was transferred back to the ICU and was intubated for airway protection. Upper endoscopy on hospital day 5 showed food intermixed with coffee-groundlike content in the gastric fundus and body. A $6 \mathrm{~mm}$ ulcer with a visible vessel was identified in the greater curvature (Fig. 1B) without any evidence of active bleeding. Epinephrine $(3 \mathrm{~mL}$ of $1: 10,000)$ was injected around the ulcer, and three endoclips were placed to prevent bleeding. However, the same evening, the patient's hemodynamics worsened, and his family withdrew care. He died the same night.

\section{Case 3}

A 76-year-old Caucasian man with a medical history of type 2 diabetes mellitus, hypertension, hyperlipidemia, and prostate cancer, presented to the hospital owing to progressive symptoms of COVID-19, who was being previously managed conservatively on an outpatient basis. He initially visited the emergency department with complaints of headache, nausea, and dizziness, and he had come in contact with multiple people 
who were recently diagnosed with COVID-19 (via RT-PCR testing). He developed diarrhea, persistent fever, and progressive shortness of breath over the next 4 days and returned to the hospital for evaluation. He was started on dexamethasone for COVID-19, along with ceftriaxone and azithromycin for community-acquired pneumonia. He was transferred to the ICU owing to worsening dyspnea, requiring intubation on hospital day 2. He self-extubated on hospital day 4 and was eventually transferred to the medicine floor. On hospital day 11 , he was started on weight-based enoxaparin for bilateral pulmonary emboli. Shortly after initiating anticoagulation therapy, the patient was transferred back to the ICU owing to hematemesis and for airway protection. He was re-intubated and underwent upper endoscopy on hospital day 12 because of a significant drop in hemoglobin levels and subsequent hypotension. An $8 \mathrm{~mm}$ vessel was seen within an ulcer in the distal esophagus without active bleeding (Fig. 1C). Three clips were placed for hemostasis with good effect. Solid food intermixed with coffee-ground-like content was observed in the gastric body. The patient was extubated the following day and ultimately discharged home on hospital day 23 .

\section{Case 4}

A 69-year-old Caucasian man with a medical history of hypertension, hyperlipidemia, and obstructive sleep apnea was transferred to our center with shortness of breath, fever, and cough for 3 weeks. He was diagnosed with COVID-19 10 days prior to transfer, which was confirmed by RT-PCR testing. The patient was treated with ceftriaxone and azithromycin for community-acquired pneumonia, and dexamethasone and remdesivir for COVID-19. He initially required a non-rebreather mask for oxygenation, which was transitioned to a high-flow nasal cannula and eventually to a standard nasal cannula. The patient was found to have new pulmonary emboli on hospital day 6 and was started on weight-based enoxaparin. On hospital day 10, a rapid response was called owing to hematemesis along with hypotension. There was no known history of liver disease or the use of NSAIDs or antiplatelet agents. The patient was transferred to the ICU and was subsequently intubated for airway protection. Owing to concerns of hemorrhagic shock, urgent endoscopy was conducted, revealing large amounts of freshly clotted blood in the fundus and body of the stomach. Multiple ulcerations were noted in the antrum and incisura, ranging from 3 to $10 \mathrm{~mm}$ in size (Fig. 1D). Two ulcers were oozing from the edges, and three endoclips were placed for hemostasis. The rest of the ulcers were clean based. No further episodes of bleeding occurred. The patient has since required a tracheostomy and remains in the hospital for recovery at the time of writing.

\section{DISCUSSION}

COVID-19 has been associated with a myriad of symptoms, and patients who require hospitalization can have a severe and prolonged course. GI bleeding has been observed in this patient population, and recent case series ${ }^{1,2}$ have described good outcomes with conservative management. We presented four cases of patients with a severe COVID-19 disease course, requiring intubation, with significant GI bleeding necessitating urgent endoscopic evaluation (Table 1). Although Lau et al. ${ }^{3}$ demonstrated that delaying endoscopy for $24 \mathrm{hr}$ did not lead to an increased 30-day mortality risk, given the acuity of hemoglobin changes and concerns for hemorrhagic shock in all four cases, endoscopy was conducted within $24 \mathrm{hr}$ of GI consultation, despite the COVID-19 exposure risk.

In the cases described, all patients were critically ill and had multi-organ complications. Two of these cases developed pulmonary emboli requiring the use of weight-based enoxaparin. This medication needed to be withheld for the endoscopic evaluation of GI bleeding, another factor in favor of early endoscopic visualization. In both cases, the patients were able to resume anticoagulation within days of the endoscopy without recurrent bleeding. The procoagulant complications of COVID-19 are being increasingly identified, ${ }^{4}$ and in patients with GI bleeding, endoscopy findings may factor in the decision-making process. In both the patients who developed pulmonary emboli, endoscopic findings indicated that intervention (visible vessels requiring endoclips) is required, and we postulate that this helped resume systemic anticoagulation in a timely manner. Thus, both patients were alive at the time of writing.

Although critically ill patients in the ICU have an increased risk of GI bleeding in general, whether COVID-19, as an independent risk factor, truly portends a high mortality rate is an important topic for future studies, or risk stratification models for goals of care discussions. It is difficult to say whether COVID-19 carries an independent risk of bleeding, or if the bleeding represents sequelae from systemic stress related to severe acute illness. COVID-19 utilizes angiotensin-converting enzyme II receptors for cell entry, but it remains unclear whether this mechanism leads to bleeding diathesis. ${ }^{5}$

Endoscopy is considered an aerosolizing procedure for patients with COVID-19, and the benefits of endoscopic evaluation and potential treatment should be carefully weighed against the potential infectious risks to physicians, nurses, and technicians. None of the GI staff involved with the endoscopies were infected, as appropriate personal protective equipment (face shield, double gloves, N95 masks or powered air-purifying respirators, gowns, shoe covers, and hair covering) were donned and doffed for the procedures. 
Table 1. Patient Demographics and Clinical Factors

\begin{tabular}{|c|c|c|c|c|}
\hline & Patient 1 & Patient 2 & Patient 3 & Patient 4 \\
\hline Age (yr) & 69 & 68 & 76 & 69 \\
\hline Gender & Male & Male & Male & Male \\
\hline Ethnicity & Caucasian & African-American & Caucasian & Caucasian \\
\hline Prior history GI bleed & No & Yes & No & No \\
\hline COVID-19 treatment & $\begin{array}{c}\text { Dexamethasone } \\
\text { Convalescent plasma }\end{array}$ & Dexamethasone & Dexamethasone & $\begin{array}{l}\text { Dexamethasone } \\
\text { Remdesivir }\end{array}$ \\
\hline Intubated for endoscopy? & No, already intubated & Yes, and airway protection & Yes, and airway protection & Yes, and airway protection \\
\hline Hemodynamic instability? & Yes & Yes & Yes & Yes \\
\hline Glasgow-Blatchford score & 10 & 20 & 12 & 13 \\
\hline $\begin{array}{l}\text { PPI or H2-blocker use within } \\
48 \text { hours prior to bleeding }\end{array}$ & Yes & Yes & No & No \\
\hline Laboratory results & - & - & - & - \\
\hline Baseline Hb (g/dL)/HCT (\%) & $10.4 / 31.0$ & $10.3 / 31.9$ & $12.2 / 35.1$ & $11.7 / 31.9$ \\
\hline Lowest $\mathrm{Hb}$ (g/dL)/HCT (\%) & $5.9 / 17.7$ & $6.0 / 19.2$ & $9.0 / 26.7$ & $8.4 / 23.3$ \\
\hline PT (sec)/INR (IU) & $1.0 / 33.2$ & $1.3 / 15.7$ & $1.2 / 14.8$ & $1.3 / 16.5$ \\
\hline BUN (mg/dL)/Cr (mg/dL) & $46 / 2.62$ & $72 / 7.73$ & $76 / 1.95$ & $32 / 0.92$ \\
\hline Platelet count $(\mathrm{K} / \mathrm{uL})$ & 125 & 100 & 340 & 351 \\
\hline Units of pRBC & 3 & 2 & 0 & 0 \\
\hline Systemic anticoagulation? & No & No & Yes & Yes \\
\hline
\end{tabular}

Clinical outcome Died, unrelated to bleed Died, unrelated to bleed Discharged Remains in ICU

BUN, blood urea nitrogen; COVID-19, coronavirus disease; Cr, creatinine; GI, gastrointestinal; Hb, hemoglobin; HCT, hematocrit; ICU, intensive care unit; INR, international normalized ratio; PPI, proton pump inhibitor; pRBC, packed red blood cells; PT, prothrombin time.

In conclusion, in patients with COVID-19 who develop hemodynamically significant upper GI bleeding, endoscopic evaluation may be beneficial; however, in our small patient cohort, significant mortality was noted. Goals of care discussions, weighing risks with potential benefits of endoscopy, should be undertaken. Future research should focus on whether various treatment regimens for COVID-19 lead to increased GI bleeding.

\section{Conflicts of Interest}

The authors have no potential conflicts of interest.

Funding

None.

ORCID

Faruq Pradhan: $\quad$ https://orcid.org/0000-0002-5249-188X

Yasmin Alishahi: https://orcid.org/0000-0001-7379-0654

\section{REFERENCES}

1. Gadiparthi C, Perisetti A, Sayana H, Tharian B, Inamdar S, Korman A. Gastrointestinal bleeding in patients with severe SARS-CoV-2. Am J Gastroenterol 2020;115:1283-1285.

2. Cavaliere K, Levine C, Wander P, Sejpal DV, Trindade AJ. Management of upper GI bleeding in patients with COVID-19 pneumonia. Gastrointest Endosc 2020;92:454-455.

3. Lau JYW, Yu Y, Tang RSY, et al. Timing of endoscopy for acute upper gastrointestinal bleeding. N Engl J Med 2020;382:1299-1308.

4. Ranucci M, Ballotta A, Di Dedda U, et al. The procoagulant pattern of patients with COVID-19 acute respiratory distress syndrome. J Thromb Haemost 2020;18:1747-1751.

5. Wu F, Zhao S, Yu B, et al. A new coronavirus associated with human respiratory disease in China. Nature 2020;579:265-269. 